\title{
Consenso de Lúpus Eritematoso Sistêmico
}

\section{Consensus of Systemic Lupus Erythematosus}

\author{
Eduardo Ferreira Borba ${ }^{(1)}$, Luiz Carlos Latorre ${ }^{(2)}$, João Carlos Tavares Brenol ${ }^{(3)}$, Cristiane Kayser ${ }^{(4)}$, \\ Nilzio Antonio da Silva ${ }^{(5)}$, Adriana Fontes Zimmermann ${ }^{(6)}$, Paulo Madureira de Pádua ${ }^{(7)}$, \\ Lilian Tereza Lavras Costallat ${ }^{(8)}$, Eloísa Bonfá( ${ }^{(9)}$, Emília Inoue Sato $^{(10)}$
}

\section{DESCRIÇÃO DO MÉTODO DE COLETA DE EVIDÊNCIAS}

Dez reumatologistas que trabalham em serviços que atendem grande número de pacientes com lúpus eritematoso sistêmico, alguns dos quais têm pesquisa e publicações científicas nesta área, foram convidados a participar do grupo de trabalho. Todos se reuniram para discutir o tratamento das diferentes manifestações da doença, subdivididos em grupos de trabalho, cada qual ficando responsável por buscar a melhor evidência para o tratamento de um ou mais comprometimentos da doença. A última edição de Dubois's Lupus Erythematosus, editado por Wallace D e Hahn B, em 2007 (Lippincott Williams \& Wilkins), foi utilizada como base da discussão. Trabalhos publicados nos últimos cinco anos foram pesquisados no MedLine. Em virtude da freqüência e da heterogeneidade de manifestações da doença, a maioria dos trabalhos terapêuticos não contempla grande casuística, nem são randômicos e controlados. Como as manifestações e a gravidade da doença variam em diferentes grupos populacionais, devemos avaliar com cuidado os estudos realizados em grupos populacionais distintos.

\section{GRAU DE RECOMENDAÇÃO E FORÇA DE EVIDÊNCIA}

A: Estudos experimentais e observacionais de melhor consistência.

B: Estudos experimentais e observacionais de menor consistência.

C: Relatos de casos (estudos não controlados).
D: Opinião desprovida de avaliação crítica, com base em consensos, estudos fisiológicos ou modelos animais.

\section{OBJETIVOS}

Elaborar recomendação fundamentada na melhor evidência científica para o tratamento das diversas manifestações do lúpus eritematoso sistêmico.

\section{INTRODUÇÃO}

O lúpus eritematoso sistêmico (LES) é uma doença inflamatória crônica, multissistêmica, de causa desconhecida e de natureza auto-imune, caracterizada pela presença de diversos auto-anticorpos. Evolui com manifestações clínicas polimórficas, com períodos de exacerbações e remissões. De etiologia não totalmente esclarecida, o desenvolvimento da doença está ligado a predisposição genética e fatores ambientais, como luz ultravioleta e alguns medicamentos.

É uma doença rara, incidindo, mais freqüentemente, em mulheres jovens, ou seja, na fase reprodutiva, na proporção de nove a dez mulheres para um homem, e com prevalência variando de 14 a 50/100.000 habitantes, em estudos norte-americanos ${ }^{(1)}(\mathbf{D})$. A doença pode ocorrer em todas as raças e em todas as partes do mundo.

$\mathrm{Na}$ prática, para o diagnóstico de LES utilizam-se os critérios de classificação propostos pelo American College of Rheumatology ${ }^{(2)}(\mathbf{B})$, em 1982, e revisados em $1997^{(3)}(\mathbf{D})$. O diagnóstico se fundamenta na presença de, pelo menos, quatro dos 11 critérios descritos na Tabela 1.

\footnotetext{
Declaramos a inexistência de conflitos de interesse.

1. Professor-Assistente da Disciplina de Reumatologia da Faculdade de Medicina da Universidade de São Paulo (FMUSP).

2. Diretor dos Serviços de Clínicas Médicas do Hospital Heliópolis de São Paulo.

3. Professor de Reumatologia da Faculdade de Medicina da Universidade Federal do Rio Grande do Sul

4. Médica-assistente da Disciplina de Reumatologia da Universidade Federal de São Paulo (UNIFESP) - Escola Paulista de Medicina

5. Professor Titular de Reumatologia da Faculdade de Medicina da Universidade Federal de Goiás

6. Médica Reumatologista do Hospital Universitário e Professora da Disciplina de Reumatologia da Universidade Federal de Santa Catarina (UFSC).

7. Titular de Reumatologia da Faculdade de Medicina da Universidade Federal de Minas Gerais (FMUFMG), Coordenador da Clínica Reumatológica da Santa Casa de Belo Horizonte, MG.

8. Professora Titular de Reumatologia da Faculdade de Ciências Médicas da Universidade Estadual de Campinas (Unicamp)

9. Professora Titular da Disciplina de Reumatologia da Faculdade de Medicina da USP.

10. Professora Titular de Reumatologia da Universidade Federal de São Paulo (UNIFESP).

Endereço para correspondência: Sociedade Brasileira de Reumatologia. Av. Brigadeiro Luís Antônio, 2.466, cj. 93-94 - 01402-000 - São Paulo - SP, fone/fax: 55 (11) 3289-7165. E-mail:sbre@terra.com.br
} 
TABELA 1

Critérios de ClassificaÇão de LES do American College of Rheumatology Revisados em $1997^{3}$

1. Eritema malar: lesão eritematosa fixa em região malar, plana ou em relevo.

2. Lesão discóide: lesão eritematosa, infiltrada, com escamas queratóticas aderidas e tampões foliculares, que evolui com cicatriz atrófica e discromia.

3. Fotossensibilidade: exantema cutâneo como reação não-usual à exposição à luz solar, de acordo com a história do paciente ou observado pelo médico.

4. Úlceras orais/nasais: úlceras orais ou nasofaríngeas, usualmente indolores, observadas pelo médico.

5. Artrite: não-erosiva envolvendo duas ou mais articulações periféricas, caracterizadas por dor e edema ou derrame articular.

6. Serosite: pleuris (caracterizada por história convincente de dor pleurítica, atrito auscultado pelo médico ou evidência de derrame pleural) ou pericardite (documentado por eletrocardiograma, atrito ou evidência de derrame pericárdico)

7. Comprometimento renal: proteinúria persistente $(>0,5 \mathrm{~g} /$ dia ou $3+)$ ou cilindrúria anormal.

8. Alterações neurológicas: convulsão (na ausência de outra causa) ou psicose (na ausência de outra causa).

9. Alterações hematológicas: anemia hemolítica ou leucopenia (menor que $4.000 / \mathrm{mm}^{3}$ em duas ou mais ocasiões) ou linfopenia (menor que $1.500 / \mathrm{mm}^{3}$ em duas ou mais ocasiões) ou plaquetopenia (menor que $100.000 / \mathrm{mm}^{3}$ na ausência de outra causa).

10. Alterações imunológicas: anticorpo anti-DNA nativo ou anti-Sm ou presença de anticorpo antifosfolípide com base em:

a) níveis anormais de $\operatorname{IgG}$ ou $\operatorname{IgM}$ anticardiolipina;

b) teste positivo para anticoagulante lúpico; ou

c) teste falso-positivo para sífilis, por, no mínimo, seis meses.

11. Anticorpos antinucleares: título anormal de anticorpo antinuclear por imunofluorescência indireta ou método equivalente, em qualquer época, e na ausência de drogas conhecidas por estarem associadas à síndrome do lúpus induzido por drogas.

Estes critérios foram desenvolvidos com o objetivo de uniformizar os estudos científicos da doença. A avaliação laboratorial pode auxiliar sobremaneira o diagnóstico por ocasião da constatação de alterações hematológicas (leucopenia e/ ou linfopenia e/ou plaquetopenia e/ou anemia hemolítica) e alterações do sedimento urinário. Embora raro, é possível se ter pacientes com lúpus que não apresentem quatro dos critérios de classificação, principalmente quando apresentam anticorpo específico de LES (anti-DNA nativo em títulos moderados/ altos ou anti-Sm) e apenas uma manifestação clínica.

De particular importância para o diagnóstico é a pesquisa de anticorpos ou fatores antinucleares por imunofluorescência indireta, utilizando como substrato as células HEp-2, conforme proposta do II Consenso Brasileiro sobre Laudos de $\operatorname{FAN}^{(4)}(\mathbf{D})$. A positividade desse teste, embora não-específico, serve como triagem em razão de sua sensibilidade (maior que 95\%), sendo altamente improvável a presença da doença se o teste resultar negativo ${ }^{(2)}(\mathbf{B})$. A pesquisa de anticorpos como anti-DNA nativo, anti-Sm e antinucleosomo pode contribuir para melhor caracterização laboratorial do quadro. Nos raros casos da doença com pesquisa de FAN negativa, particularmente com lesões cutâneas fotossensíveis, recomenda-se a realização da pesquisa de anticorpos anti-Ro/SSa.

\section{MEDIDAS GERAIS}

Como parte importante da abordagem terapêutica inicial, algumas medidas gerais são recomendadas, entre $\operatorname{elas}^{(5)}(\mathbf{D})$ :

1. Educação: informar ao paciente e a seus familiares sobre a doença e sua evolução, possíveis riscos e os recursos disponíveis para diagnóstico e tratamento. Recomenda-se a necessidade de cumprimento das medidas estabelecidas pelo médico ${ }^{(5)}(\mathbf{D})$.

2. Apoio psicológico: transmitir otimismo e motivação para o tratamento, além de estimular os projetos de $\operatorname{vida}^{(5)}(\mathbf{D})$.

3. Atividade física: repouso nos períodos de atividade sistêmica da doença deve ser recomendado ${ }^{(5)}(\mathbf{D})$. Por causa da redução da capacidade aeróbica ${ }^{(6)}(\mathbf{B}),{ }^{(7)}(\mathbf{B})$, medidas visando a melhora do condicionamento físico devem ser estimuladas ${ }^{(8)}(\mathbf{B})$. A atividade física regular reduz risco cardiovascular e promove melhora da fadiga e da qualidade de vida ${ }^{(8)}(\mathbf{B}),{ }^{(9)}(\mathbf{B}),{ }^{(10)}(\mathbf{B})$.

4. Dieta: não há evidência científica de que os alimentos possam influenciar o desencadeamento ou a evolução da doença. Recomenda-se a adoção de dieta balanceada, evitando-se excessos de sal, carboidratos e lipídios. Déficit de vitamina D pode ocorrer com a fotoproteção e o uso de antimaláricos, e também nos quadros renais ${ }^{(11)}(\mathbf{B}),{ }^{(12)}(\mathbf{B})$. A suplementação da vitamina $\mathrm{D}$ deve ser considerada em todos os pacientes $^{(5)}(\mathbf{D})$.

5. Proteção: contra luz solar e outras formas de irradiação ultravioleta.

6. Evitar tabagismo: além de ser fator de risco para aterosclerose, diminui a eficácia dos antimaláricos ${ }^{(13)}$ $(\mathbf{B}),{ }^{(14)}(\mathbf{B})$, favorecendo a manutenção ou a piora das lesões cutâneas ${ }^{(15)}(\mathbf{B})$.

7. Controle rigoroso dos fatores de risco cardiovascular: glicemia, hipertensão arterial, dislipidemia e obesidade $^{(16)}(\mathbf{B})$.

\section{TRATAMENTO MEDICAMENTOSO}

O tratamento medicamentoso deve ser individualizado para cada paciente e depende dos órgãos ou dos sistemas acometidos, bem como da gravidade destes acometimentos. 
Diante do comprometimento de múltiplos sistemas, o tratamento deverá ser orientado para o comprometimento mais grave. Quando houver manifestação que não responda à droga, pode ser necessário fazer uso concomitante de diversos medicamentos. Por exemplo, um paciente com nefrite em uso de corticosteróide e imunossupressor, mas com lesões cutâneas refratárias, pode necessitar do uso concomitante de talidomida ou dapsona.

Independentemente do órgão ou do sistema afetado, o uso contínuo de antimaláricos, preferencialmente do sulfato de hidroxicloroquina, é indicado com a finalidade de reduzir a atividade da doença e tentar poupar o uso de corticóides $^{(17)}(\mathbf{B})$. A manutenção deste medicamento em pacientes controlados reduz a possibilidade de novo surto de atividade ${ }^{(18)}(\mathbf{A})$. Melhora do perfil lipídico ${ }^{(19)}(\mathbf{B}),{ }^{(20)}$ (B) e redução do risco de trombose ${ }^{(21)}(\mathbf{D})$ são benefícios adicionais atribuídos ao uso de antimaláricos.

Além dos antimaláricos, os glicocorticóides são os fármacos mais utilizados no tratamento e as suas doses diárias variam de acordo com a gravidade de cada caso. Tendo a prednisona como padrão, didaticamente estas doses podem ser divididas $\mathrm{em}^{(22)}(\mathbf{D})$ :

- Dose baixa: $\quad 0,125 \mathrm{mg} / \mathrm{kg} / \mathrm{dia}$;

- Dose moderada: 0,125 a $0,5 \mathrm{mg} / \mathrm{kg} / \mathrm{dia}$;

- Dose alta: $\quad 0,6$ a $1 \mathrm{mg} / \mathrm{kg} /$ dia;

- Dose muito alta: 1 a $2 \mathrm{mg} / \mathrm{kg} /$ dia.

- Pulsoterapia com glicocorticóides: Aplicação intravenosa de metilprednisolona ( 15 a $20 \mathrm{mg} / \mathrm{kg} / \mathrm{dia}$ ), habitualmente por três dias consecutivos.

Em virtude de vários efeitos colaterais, os glicocorticóides devem ser utilizados na menor dose efetiva para o controle da atividade da doença e com redução gradual de sua dose, assim que possível. Embora haja grande variabilidade individual na sensibilidade aos glicocorticóides, está demonstrado que o uso de glicocorticóides de longa ação, como a dexametasona, é o mais deletério, devendo ser evitado o seu uso diário ${ }^{(23)}(\mathbf{D})$. Nos pacientes que não conseguem atingir a dose de manutenção de glicocorticóides menor ou igual a 7,5 mg/dia, está indicada a associação de outra droga para poupar glicocorticóides, além dos antimaláricos ${ }^{(17)}(\mathbf{B})$. Entre os medicamentos com comprovada ação poupadora de glicocorticóides, destacamse também a azatioprina ${ }^{(24)}(\mathbf{D})$ e o metotrexato ${ }^{(25)}(\mathbf{C})$. Os pacientes em uso de glicocorticóides necessitam de suplementação de cálcio, além da de vitamina $\mathrm{D}^{(5)}(\mathbf{D})$.

É importante o diagnóstico diferencial entre atividade da doença e infecção, ressaltando a possibilidade de coexistência de ambas, assim como da presença de comorbidades.

\section{TRATAMENTO MEDICAMENTOSO POR ACOMETIMENTOS}

\section{COMPROMETIMENTO CUTÂNEO}

O tratamento vai depender da extensão do acometimento dermatológico e da gravidade das manifestações extracutâneas. Salientar que nem toda lesão cutânea é LES específica, podendo decorrer de complicações do tratamento ou outras dermatoses concomitantes, requerendo condutas diagnósticas e terapêuticas diversas.

\section{FOTOPROTEÇÃo}

Considerando que a radiação ultravioleta B é a principal causadora de fotossensibilidade e desencadeante das lesões cutâneas, protetores solares com FPS de, no mínimo, 15 devem ser utilizados em quantidade generosa pela manhã e reaplicados mais de uma vez ao dia.

$\mathrm{O}$ uso de bloqueadores solares de amplo espectro pode trazer benefício adicional pela capacidade de proteção contra UV-A, em algumas lesões cutâneas, como as lesões subagudas $^{(26)}(\mathbf{D})$, bem como no melhor prognóstico relacionado às lesões renais e trombocitopenia ${ }^{(27)}(\mathbf{B})$.

Nas lesões cutâneas localizadas, está indicada terapia tópica com corticóide não-fluorado na face e em áreas de flexão. Em lesões mais hipertróficas, indica-se corticóide fluorado, podendo ser aplicado sob a forma oclusiva ou de infiltração ${ }^{(28)}(\mathbf{D})$. Casos refratários podem responder ao uso tópico dos inibidores da calcineurina, como o tacrolimo e o pimecrolimo ${ }^{(29)}(\mathbf{C}),{ }^{(30)}(\mathbf{C})$.

\section{TERAPIA SISTÊMICA}

Embora as lesões cutâneas agudas geralmente respondam bem ao tratamento indicado para outras manifestações do LES, na prática clínica utilizam-se os antimaláricos para o tratamento do comprometimento cutâneo, isolado ou associado às manifestações sistêmicas. As lesões do lúpus cutâneo subagudo costumam responder bem ao uso de antimaláricos isolados ou em combinação com a terapia tópica $^{(31)}(\mathbf{D})$. Na falta de resposta em três meses, ou antes, quando a lesão for muito extensa, ou quando houver progressiva piora, pode-se associar prednisona em dose baixa a moderada por curto período. Nos pacientes que mantêm lesão cutânea, pode-se associar metotrexato na dose de 10 a $20 \mathrm{mg} / \operatorname{semana}^{(25)}(\mathbf{A}),{ }^{(32)}(\mathbf{C})$, azatioprina ${ }^{(33)}(\mathbf{C}),{ }^{\left({ }^{(3)}\right)}(\mathbf{A})$, clofazimine $^{(35)}(\mathbf{A})$ ou dapsona, indicada principalmente para 
casos de lúpus bolhoso ${ }^{(36)}(\mathbf{D}),{ }^{(37)}(\mathbf{C})$. A talidomida pode ser indicada nos casos de lesões cutâneas refratárias, mas seu uso é proibido em mulheres férteis ${ }^{(38)}(\mathbf{B}){ }^{(39)}(\mathbf{B})$. O uso do micofenolato mofetil nos casos cutâneos refratários ainda é controverso ${ }^{(40)}(\mathbf{B}),{ }^{(41)}(\mathbf{C})$.

\section{COMPROMETIMENTO ARTICULAR}

A artrite habitualmente é intermitente e não-erosiva, entretanto, cerca de $10 \%$ dos casos podem evoluir com poliartrite ou oligoartrite crônica. As artrites agudas, quando não acompanhadas de comprometimento sistêmico, podem ser tratadas com antiinflamatórios não-hormonais, desde que não sejam contra-indicados ${ }^{(42)}(\mathbf{D})$. Caso não haja melhora, pode-se substituir ou associar prednisona em dose baixa.

Nas artrites com evolução crônica ou com recidivas freqüentes, não controladas com uso de antimalárico ${ }^{(43)}$ (A) pode-se associar metotrexato ${ }^{(44)}(\mathbf{B})$.

$\mathrm{Na}$ prática, em pacientes que evoluem com artrite crônica, em uma ou duas articulações, não-responsivos a tratamento medicamentoso antes proposto, pode-se fazer infiltração intra-articular com glicocorticóide de depósito, geralmente com boa resposta, mas não há nenhum estudo controlado que tenha avaliado esta conduta.

\section{COMPROMETIMENTO HEMATOLÓGICO}

Não há estudos controlados avaliando o tratamento das anemias hemolíticas auto-imunes (AHAI). Acrescente-se a essa dificuldade a freqüente associação desta manifestação a outros comprometimentos graves, cujo tratamento pode dificultar a avaliação mais precisa da melhor abordagem do tratamento da AHAI. O tratamento de escolha é feito com prednisona em dose alta, ou muito alta, por 4 a 6 semanas com posterior redução, na dependência da resposta. Setenta e cinco por cento dos casos respondem satisfatoriamente a esta terapêutica ${ }^{(45)}(\mathbf{D}),{ }^{(46)}(\mathbf{D})$. A pulsoterapia com metilprednisolona deve ser indicada para casos graves, em que se requer resposta mais rápida e a manutenção é feita com prednisona por via oral em dose baixa ${ }^{(47)}(\mathbf{D})$.

Nos casos refratários à corticoterapia, ou por ocasião da necessidade de altas doses de manutenção, pode-se associar imunossupressores $^{(48)}(\mathbf{D}),{ }^{(49)}(\mathbf{D})$, como a azatioprina ${ }^{(48)}(\mathbf{D})$, o micofenolato de mofetil ${ }^{(50)}(\mathbf{D})$, a ciclosporina ${ }^{(51)}(\mathbf{D})$ ou o danazol ${ }^{(46)}(\mathbf{D}),{ }^{(49)}(\mathbf{D}),{ }^{(52)}(\mathbf{D})$. Pode ser indicada também administração de anti-CD20, sobretudo nos casos de risco maior $^{(53)}(\mathbf{D})$. A imunoglobulina intravenosa mostrou ser eficaz em pacientes com anemia hemolítica auto-imune, podendo ser indicada em casos muito específicos, como os que têm contra-indicação ou toxicidade com outras terapias $^{(54)}(\mathbf{C})$.

Em relação a leucopenias, o tratamento com prednisona deve ser instituído quando a contagem de leucócitos for inferior a $2.000 / \mathrm{mL}$. Em situações de grave neutropenia pode ser prescrito pulsoterapia com metilprednisolona associada a fator-estimulador de colônia de granulócitos ${ }^{(55)}$ $(\mathbf{C}),{ }^{(56)}(\mathbf{C})$.

As plaquetopenias leves, contagem maior que $50 \mathrm{mil}$, geralmente não requerem tratamento específico ${ }^{(45)}(\mathbf{D})$. A prednisona em dose alta é o tratamento de escolha para plaquetopenia sintomática. A pulsoterapia com metilprednisolona pode ser utilizada para plaquetopenia grave, quando se requer resposta mais rápida, embora sua superioridade em relação ao uso de glicocorticóides oral não tenha sido confirmada $^{(45)}(\mathbf{D})$. Na falta de resposta aos glicocorticóides, danazol, um esteróide androgênico com poucos efeitos virilizantes pode ser utilizado ${ }^{(57)}(\mathbf{A})$. Outras drogas que têm mostrado alguma eficácia em pequeno número de casos com trombocitopenia refratária são: ciclofosfamida ${ }^{(58)}(\mathbf{C})$, antimaláricos associados à prednisona ${ }^{(59)}(\mathbf{C})$, azatioprina ${ }^{(60)}$ $(\mathbf{C})$, micofenolato de mofetil ${ }^{(61)}(\mathbf{D})$ e ciclosporina ${ }^{(62)}(\mathbf{D})$. A administração de anti-CD20 também pode ser indicada em casos graves ${ }^{(63)}(\mathbf{D}),{ }^{(64)}(\mathbf{D})$. Imunoglobulina intravenosa pode ser indicada ${ }^{(65)}(\mathbf{C})$ associada à infusão de plaquetas ${ }^{(66)}$ (C) nas plaquetopenias graves, com risco de morte. Seu custo extremamente alto deve ser considerado. A efetividade da esplenectomia em trombocitopenia refratária a glicocorticóides é controversa. Um relato de caso mostrou resposta favorável e persistente ${ }^{(59)}(\mathbf{C})$, no entanto, a esplenectomia só deve ser indicada depois da falha de outras terapias medicamentosas ${ }^{(45)}(\mathbf{D}),{ }^{(67)}(\mathbf{D})$.

\section{COMPROMETIMENTO CARDÍACO}

Casos leves de pericardite podem apresentar resposta a antiinflamatórios não-hormonais (AINH) e formas mais graves geralmente respondem à corticoterapia em doses moderadas $^{(68)}(\mathbf{D})$. A pericardiocentese é indicada nos casos de tamponamento cardíaco ${ }^{(68)}(\mathbf{D})$. A corticoterapia em alta dose, oral ou em pulsos endovenosos, é necessária nas fases iniciais de miocardite. Em casos refratários, utilizamse imunossupressores (azatioprina ou ciclofosfamida) ou gamaglobulina ${ }^{(68)}(\mathbf{D})$. A endocardite de Libman-Sacks não necessita de tratamento específico com exceção dos casos com comprometimento hemodinâmico ${ }^{(68)}(\mathbf{D})$. 


\section{ATEROSCLEROSE}

A aterosclerose assume grande importância na doença, pois contribui diretamente para a sua maior morbimortalidade ${ }^{(68)}(\mathbf{D}),{ }^{69}(\mathbf{A})$. O processo é precoce e agravado pelo uso crônico de corticosteróides, hipertensão arterial, menopausa precoce e dislipidemias ${ }^{(69)}(\mathbf{A})$. As lesões endoteliais e a inflamação favorecem a formação da placa aterosclerótica $^{(69)}(\mathbf{A})$. Por causa do maior risco de infarto agudo do miocárdio ( 7 vezes maior que a população normal $)^{(70)}(\mathbf{A})$, existe a necessidade de controlar os fatores de risco cardiovascular associados, como citado previamente ${ }^{(69)}$ (A). Os antimaláricos promovem redução do colesterol $\operatorname{total}^{(71)}(\mathbf{B})$ e da $\operatorname{LDL}^{(72)}(\mathbf{B})$, além de elevação dos níveis da $\operatorname{HDL}^{(20)}(\mathbf{B})$. As estatinas podem ser utilizadas para o controle das dislipidemias, pois também promovem redução da espessura médio-intimal ${ }^{(72)}(\mathbf{B})$ e melhora da função endotelial $^{(73)}(\mathbf{B})$.

\section{COMPROMETIMENTO PULMONAR}

Os antiinflamatórios não-hormonais (AINH) podem ser utilizados para o tratamento de casos leves de pleurite ${ }^{(74)}(\mathbf{D})$. Os corticosteróides em doses moderadas são indicados para casos mais graves ou que não responderam ao tratamento inicial com $\mathrm{AINH}^{(75)}(\mathbf{C})$. A azatioprina pode ser utilizada em casos não-responsivos ${ }^{(74)}(\mathbf{D})$. A pleurectomia, ou a pleurodese, é indicada para casos refratários ${ }^{(76)}(\mathbf{C}),{ }^{(77)}(\mathbf{C})$.

A pneumonite aguda e a hemorragia pulmonar são manifestações raras, nas quais a pulsoterapia com corticosteróides isolada ou em combinação com ciclofosfamida endovenosa/ oral são preconizadas ${ }^{(76)}(\mathbf{C}),{ }^{(78)}(\mathbf{C})$. A plasmaferese também é indicada para o tratamento da hemorragia pulmonar embora os seus resultados sejam controversos ${ }^{(76)}(\mathbf{C})$. A ciclofosfamida oral ou em pulsoterapia mensal, associada ou não a corticosteróides, costuma levar à estabilização ou à melhora da doença pulmonar intersticial ${ }^{(79)}(\mathbf{C}),{ }^{(80)}(\mathbf{C})$.

$\mathrm{Na}$ hipertensão pulmonar, a anticoagulação oral é recomendada por causa do maior risco de trombose in situ(76) (D). Os bloqueadores de canais de cálcio são indicados apenas para aqueles que respondem ao teste de vasorreatividade aguda durante o cateterismo ${ }^{(76)}(\mathbf{D})$. Novas drogas vasoativas, como os análogos da prostaciclina (epoprostenol, treprostinil) ${ }^{(81)}(\mathbf{B})$, o bosentan (antagonista dos receptores da endotelina $)^{(82)}(\mathbf{B})$ e o sildenafil (inibidor da fosfodiesterase $5)^{(83)}(\mathbf{B})$, podem ser consideradas como opção terapêutica. Uma pequena porcentagem dos pacientes apresenta melhora da hipertensão pulmonar com o uso de corticosteróides e pulsoterapia com ciclofosfamida ${ }^{(76)}(\mathbf{C}),{ }^{(84)}(\mathbf{C})$.

\section{COMPROMETIMENTO NEUROPSIQUIÁTRICO}

O espectro das manifestações neuropsiquiátricas é muito variável, podendo acometer os sistemas nervosos central e periférico. Em 1999, o subcomitê do Colégio Americano de Reumatologia classificou 19 síndromes neuropsiquiátricas relacionadas à doença lúpica $^{(85)}(\mathbf{D})$ (Tabela 2).

TABELA 2

SÍNDROMES NEUROPSIQUIÁTRICAS RELACIONADAS AO LÚPUS ERITEMATOSO SISTÊMICO ${ }^{(85)}$

\begin{tabular}{l}
\hline Sistema nervoso central \\
Estado confusional agudo \\
Distúrbios cognitivos \\
Psicose \\
Desordens de humor \\
Desordens de ansiedade \\
Cefaléia \\
Doença cerebrovascular \\
Mielopatia \\
Desordens do movimento \\
Síndromes desmielinizantes \\
Convulsões \\
Meningite asséptica \\
Sistema nervoso periférico \\
Neuropatia craniana \\
Polineuropatia \\
Plexopatia \\
Mononeuropatia simples/múltipla \\
Polirradiculoneuropatia inflamatória aguda (Guillain-Barré) \\
Desordens autonômicas \\
Miastenia grave
\end{tabular}

O tratamento das manifestações neuropsiquiátricas deverá ser dirigido ao tipo de manifestação apresentada. Embora não haja estudos terapêuticos controlados, podem ser tratadas com glicocorticóides e/ou imunossupressores, preferencialmente a ciclofosfamida ${ }^{(86)}(\mathbf{C})$, em doses que variam de acordo com a gravidade.

Nos casos de doenças cerebrovasculares, em decorrência de fenômenos tromboembólicos, muitas vezes relacionados aos anticorpos antifosfolípides, o uso de anticoagulantes está indicado $^{(87)}(\mathbf{D})$. Para o tratamento das convulsões podem ser prescritos anticonvulsivantes, assim como na psicose os antipsicóticos podem ser bons coadjuvantes ${ }^{(87)}(\mathbf{D})$.

A mononeuropatia e a polineuropatia periférica geralmente respondem ao esquema de corticosteróides e/ou imunossupressores $^{(87)}(\mathbf{D})$. A neuropatia craniana ocorre, em geral, em doença ativa, é transitória e responde ao tratamento convencional com glicocorticóides em altas $\operatorname{doses}^{(87)}(\mathbf{D})$. 
A mielopatia deve ser tratada com pulsoterapia de corticosteróides associada à ciclofosfamida endovenosa ${ }^{(88)}(\mathbf{B})$.

Nos casos com comprometimento neurológico grave não-responsivos às medidas anteriormente citadas, pode-se indicar imunoglobulina endovenosa ou plasmaferese, embora os resultados sejam controversos ${ }^{(87)}(\mathbf{D})$. O rituximabe (anti-CD-20) pode também ser uma opção para os casos refratários $^{(89)}(\mathbf{D})$.

\section{COMPROMETIMENTO RENAL}

Os principais objetivos do tratamento da nefropatia são controlar a atividade inflamatória e prevenir a evolução para a insuficiência renal crônica. Os parâmetros utilizados para avaliar o envolvimento renal e o monitoramento da terapêutica são ${ }^{(90)}(\mathbf{D})$ :

- clínico: edema, oligúria e hipertensão arterial; em número significativo de casos pode ser assintomática;

- laboratorial: exame do sedimento urinário, proteinúria de 24 horas, creatinina e albumina séricas, depuração de creatinina, C3 e anti-dsDNA;

- biópsia renal: é recomendável sua realização em pacientes com envolvimento renal, caracterizado por sedimento urinário anormal (cilindrúria) e/ou proteinúria maior que $0,5 \mathrm{~g} / 24$ horas e/ou alteração da função renal, excluindo-se sua indicação na nefropatia crônica terminal. Este procedimento poderá melhor orientar o tratamento e o prognóstico, devendo ser realizado por profissionais experientes e habilitados ${ }^{(91)}$ (D). O padrão histológico de glomerulonefrite lúpica deve seguir as novas definições recentemente revisadas pelas sociedades internacionais de nefrologia e patologia (classificação da ISN/RPS 2004) $)^{(92)}(\mathbf{A}),{ }^{(93)}(\mathbf{A})$.

\section{CRITÉRIOS DE RESPOSTA À TERAPIA}

Recentemente, o Colégio Americano de Reumatologia publicou recomendações mínimas para avaliar a resposta terapêutica na nefrite lúpica ${ }^{(94)}(\mathbf{A})$. Variáveis clínicas (idade, raça, sexo, tempo de doença, comorbidades etc.), laboratoriais (clearence de creatinina, proteinúria, sedimento urinário etc.) e padrão his-

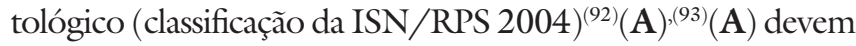
fazer parte da interpretação de resposta terapêutica na nefrite lúpica. Essa avaliação deve ser feita após o período mínimo de seis meses e permite melhor interpretação após um ano, mas, idealmente, deve ser considerada após cinco $\operatorname{anos}^{(94)}(\mathbf{A})$.

\section{Medidas gerais NA Nefropatia}

1. Controle rigoroso da hipertensão arterial, estando também indicados os agentes inibidores da angio- tensina, tendo em vista seus efeitos antiproteinúricos e renoprotetores, exceto quando ocorrer disfunção renal aguda ${ }^{(95)}(\mathbf{A})$.

2. Controle da obesidade, dislipidemia e restrição ao uso de antiinflamatórios não-esteróides e outras drogas potencialmente nefrotóxicas ${ }^{(96)}(\mathbf{D})$.

\section{MEdidas MEdicamentosas}

1. Para os pacientes com glomerulonefrite proliferativas, o glicocorticóide em altas doses é a droga de escolha para a doença ativa pelo período de 6 a 8 semanas, seguindo-se sua redução progressiva ${ }^{(97)}$ (C). A pulsoterapia com metilprednisolona está indicada para os casos mais graves, com disfunção renal aguda ${ }^{(98)}(\mathbf{B})$.

2. Nas nefrites proliferativas está indicada uma fase de indução com agentes imunossupressores citostáticos (nos primeiros 6 meses), particularmente a ciclofosfamida endovenosa, inicialmente sob a forma de pulsos mensais e, posteriormente, bimestrais ou trimestrais (fase de manutenção), até um ano após a remissão da nefrite ${ }^{(99)}(\mathbf{A})$. A despeito de algumas controvérsias, até o momento, a ciclofosfamida é considerada a terapia mais efetiva para o tratamento inicial de nefrite lúpica grave, entretanto, como esta não é capaz de controlar todos os casos e em razão de sua toxicidade, novos esquemas terapêuticos devem ser avaliados ${ }^{(100)}(\mathbf{D})$. Estudos de metanálise evidenciaram a superioridade do uso associado de imunossupressores na preservação da função renal e na sobrevida dos pacientes com nefrite lúpica, quando comparado ao uso isolado de glicocorticosteróides ${ }^{(101)}(\mathbf{A})$, sem que se comprovasse superioridade de um imunossupressor sobre o outro ${ }^{(102)}(\mathbf{A})$. Em casos selecionados, pode-se utilizar o micofenolato mofetil ${ }^{(103)}(\mathbf{A})$ e o esquema seqüencial de dose baixa de ciclofosfamida e azatioprina $^{(104)}(\mathbf{A})$. Na terapia de manutenção, na tentativa de diminuir os efeitos colaterais decorrentes do uso prolongado de ciclofosfamida, pode-se utilizar azatioprina ou micofenolato mofetil, após a indução da remissão da nefrite lúpica ${ }^{(105)}(\mathbf{A})$. A opção de indução somente com micofenolato mofetil necessita maiores estudos. Recentes publicações mostram controvérsias, inclusive com respostas diferentes dependendo da raça do paciente ${ }^{(103)}$ $(\mathbf{A}),{ }^{(106)}(\mathbf{A}),{ }^{(107)}(\mathbf{A})$. 
3. Nos pacientes com nefrite membranosa pura, o tratamento é controverso. Podem ser utilizados glicocorticóides e/ou agentes imunossupressores, na dependência do quadro de síndrome nefrótica ${ }^{(108)}(\mathbf{D})$. Entre os imunossupressores podem ser indicados a ciclofosfamida oral ${ }^{(109)}(\mathbf{B})$, a ciclosporina $^{(110)}(\mathbf{C})$, a azatioprina ${ }^{(111)}(\mathbf{B})$ e o micofenolato mofetil ${ }^{(112)}(\mathbf{B})$.

4. Recentemente, vários estudos com agentes biológicos vêm sendo realizados com resultados promissores. Destacam-se como potenciais drogas para o tratamento da nefrite lúpica o rituximabe, o epratuzumabe, o belimumabe, o abetimo e o atacicepte $^{(113)}(\mathbf{C}),{ }^{(114)}(\mathbf{C})$.

5. Na situação de evolução para a perda cronicamente evolutiva da função renal, com depuração de creatinina menor que $30 \mathrm{~mL} / \mathrm{min}$, não está indicada a terapia imunossupressora, com glicocorticóides ou citostáticos, exceto para o tratamento de manifestações extra-renais.

6. A terapêutica substitutiva renal poderá incluir a utilização de métodos dialíticos e o transplante renal. A indicação deste último procedimento deverá obedecer a prazo não inferior a um ano de remissão da atividade da doença ${ }^{(115)}(\mathbf{D})$.

\section{GESTAÇÃO}

\section{FERTILIDADE E CONTRACEPÇÃO}

A fertilidade no lúpus é normal e a gravidez, embora não contra-indicada, deve ser planejada. Considerar atividade, gravidade da doença e toxicidade das drogas. A gravidez é desaconselhada quando houver atividade de doença, especialmente na vigência de nefropatia $^{(116)}(\mathbf{A})$. Gestações não planejadas devem ser evitadas com método contraceptivo efetivo. Anticoncepcionais orais de baixa dosagem de estrógenos, quando indicados, não têm mostrado indução de atividade significativa da doença, porém são contra-indicados na presença de anticorpos antifosfolípides em virtude do risco aumentado de tromboses ${ }^{(117)}(\mathbf{A}),{ }^{(118)}(\mathbf{B}),{ }^{(119)}(\mathbf{A})$.

\section{MONITORAMENTO}

O monitoramento das pacientes com LES deve ser individualizado e a gestação considerada de alto risco, necessitando acompanhamento multidisciplinar, até o puerpério, por causa da possibilidade de exacerbação da doença. Na gravidez, podem ocorrer eritemas palmar e facial, artralgias e elevação da VHS, dificultando a caracterização da atividade da doença. A presença do anti-dsDNA e/ou elevação dos seus títulos e consumo do complemento podem auxiliar na diferenciação entre atividade de doença e pré-eclâmpsia ${ }^{(120)}(\mathbf{D})$. Pacientes com anticorpos antifosfolípides têm risco maior de pré-eclâmpsia, perdas fetais, prematuridade e retardo no crescimento fetal ${ }^{(121)}(\mathbf{C})$. É importante a pesquisa dos anticorpos antifosfolípides, anti-Ro/SSA e anti-La/SSB em virtude da possibilidade da ocorrência da síndrome antifosfolípide ${ }^{(122)}(\mathbf{D})$ e do lúpus neonatal ${ }^{(123)}(\mathbf{D})$, respectivamente.

Existe maior incidência de retardo de crescimento intrauterino, prematuridade, hipertensão induzida pela gravidez e diabetes $^{(124)}(\mathbf{D})$.

\section{TERAPIA MEDICAMENTOSA NA GESTAÇÃO E AMAMENTAÇÃO}

A prednisona, a hidroxicloroquina e a ácido acetilsalićlico em baixas doses podem ser utilizadas na gravidez. Quando inevitável o uso de imunossupressores, a opção é a azatioprina $^{(125)}(\mathbf{B})$. A ciclofosfamida, o micofenolato mofetil, o metotrexato e a leflunomide não devem ser usados ${ }^{(121)}$ $(\mathbf{A}),{ }^{(126)}(\mathbf{A}),{ }^{(127)}(\mathbf{B})$. Nas gestantes com anticorpos antifosfolípides sem caracterizar a síndrome antifosfolípide utiliza-se ácido acetilsalicílico $100 \mathrm{mg} /$ dia.

$\mathrm{Na}$ amamentação, doses de glicocorticóides superiores a $20 \mathrm{mg} /$ dia de prednisona podem determinar riscos para a criança, sendo recomendado intervalo de 4 horas entre a tomada da medicação e a amamentação ${ }^{(128)}(\mathbf{C})$.

\section{SÍNDROME ANTIFOSFOLÍPIDE}

Pacientes com lúpus e anticorpos antifosfolípides com títulos moderados a altos, sem história de tromboses, podem usar ácido acetilsalicílico $100 \mathrm{mg} /$ dia por tempo indefinido. A associação com a hidroxicloroquina pode contribuir para a prevenção da trombose ${ }^{(129)}(\mathbf{C})$. Nas gestantes, nas mesmas condições e sem perdas fetais pregressas, a conduta deve ser a mesma. Nas pacientes com morbidade gestacional prévia, deve-se associar a heparina profilática durante toda a gestação. Outros fatores de risco para tromboses devem ser rigorosamente controlados (anticoncepcionais, hiperlipidemia, HAS, diabetes, fumo, sedentarismo etc.). Nos pacientes com lúpus e trombose(s) prévia(s), a anticoagulação oral deve ser feita por tempo indefinido. Nos casos de gestação, essa anticoagulação deve ser feita com heparina fracionada ou de baixo peso molecular. As únicas indicações de corticosteróides na SAF são na plaquetopenia grave e na 
síndrome catastrófica. Nesta última, gamaglobulinas EV e plasmaferese podem ser úteis ${ }^{(130)}(\mathbf{C})$.

\section{ANTICONCEPÇÃO E TERAPIA DE REPOSIÇÃO HORMONAL}

A anticoncepção pode ser feita com progesterona e métodos de barreira ou com associação de ambos. Os progestágenos podem ser utilizados, uma vez que não induzem piora da doença ${ }^{(131)}(\mathbf{B}),{ }^{(132)}(\mathbf{B})$. Os usos de anticoncepcionais orais com estrógenos em baixas doses e a terapia de reposição hormonal não promoveram aumento significativo do risco de exacerbações leves/moderadas ${ }^{(119)}$ (A). Para minimizar riscos, recomenda-se que estas terapias possam ser utilizadas em pacientes com doença inativa e sem passado de doença grave, e na ausência de anticorpos antifosfolípides, trombose prévia, tabagismo e hipertensão $\operatorname{arterial}^{(133)}(\mathbf{A}),{ }^{(134)}(\mathbf{A}),{ }^{(135)}(\mathbf{A})$.

\section{VACINAÇÃO}

As vacinas contra pneumococo ${ }^{(136)}(\mathbf{A})$ e influenza ${ }^{(137)}$ (B) são seguras e têm eficácia semelhante à da população normal. Entretanto, não devem ser administradas nos períodos de atividade da doença, no uso de quaisquer imunossupressores e nos pacientes em uso de prednisona em dose superior a $20 \mathrm{mg} / \mathrm{dia}^{(138)}(\mathbf{B})$. Nestas condições, a vacina contra hepatite $B$ parece ser segura e eficaz ${ }^{(139)}$ (B). As vacinas com vírus vivos não devem ser prescritas a pacientes com LES $\left({ }^{138)}(\mathbf{B})\right.$.

\section{REFERÊNCIAS}

1. Rus V, Maury EE, Hochberg MC: The epidemiology of systemic lupus erythematosus. In: Wallace DJ, Hahn BH, editors. Dubois lupus erythematosus. 7th ed. Philadelphia: Lippincott Williams \& Wilkins; 2007. p. 34-44.

2. Tan EM, Cohen AS, Fries JF, et al.: The 1982 revised criteria for the classification of systemic lupus erythematosus. Arthritis Rheum 25: 1271-7, 1982.

3. Hochberg MC. Updating the American College of Rheumatology revised criteria for the classification of systemic lupus erythematosus. Arthritis Rheum 40: 1725, 1997.

4. Dellavance A, Gabriel A, Cintra AFU, et al.: II Consenso Brasileiro de Fator Antinuclear em Células HEp-2. Definições para padronização da pesquisa contra constituintes do núcleo, nucléolo, citoplasma e aparelho mitótico e suas associações clínicas. Rev Bras Reumatol 43: 129-40, 2003.

5. Wallace DJ. Principles of therapy and local measures. In: Wallace DJ, Hahn BH, editors. Dubois lupus erythematosus. 7th ed. Philadelphia: Lippincott Williams \& Wilkins; 2007. p. 1132-44.

6. Tench CM, McCarthy J, McCurdie I, et al.: Fatigue in systemic lupus erythematosus: a randomized controlled trial of exercise. Rheumatology (Oxford) 42(9): 1050-4, 2003.

\section{INSTRUMENTOS DA AVALIAÇÃO}

O acompanhamento do tratamento dos pacientes com lúpus pode ser fundamentado em instrumentos que sejam capazes de medir, de maneira objetiva, a atividade inflamatória da doença (SLEDAI e SLEDAI-2K), bem como a sua seqüela (SLICC-ACR).

Medidas objetivas de atividade de doença e dano são utilizadas primariamente em ensaios clínicos e estudos observacionais ${ }^{(140)}(\mathbf{C}),{ }^{(141)}(\mathbf{C}),{ }^{(142)}(\mathbf{C})$, no entanto, podem ser utilizados na prática clínica.

O principal índice de atividade atualmente utilizado no LES é o SLEDAI (Systemic Lupus Erythematosus Disease Activity Index $)^{(143)}(\mathbf{B})$ : composto por 24 itens incluindo sinais, sintomas e testes laboratoriais, cada qual recebendo um peso (variando de 1 a 8 ), de acordo com sua importância ou gravidade. A sua soma resulta escore final, que quanto mais alto maior o grau de atividade (máximo 105). Uma modificação deste índice sem a inclusão dos testes laboratoriais é o SLEDAI-2K $\mathrm{K}^{(144)}(\mathbf{B})$. Em estudos com pacientes brasileiros com LES juvenil(145) $(\mathbf{C})$, mostrou boa aplicabilidade.

A constatação de lesão irreversível ou seqüela decorrente da doença pode ser medida por meio do SLICC/ ACR DAMAGE INDEX (SLICC/ACR: Systemic Lupus International Colaborating Clinics/ American College of Rheumatology $)^{(146)}(\mathbf{B}){ }^{(147)}(\mathbf{B})$. O índice de dano pode ficar estável ou aumentar com o decorrer do tempo e possui pontuação máxima de 47 pontos.

7. Keyser RE, Rus V, Cade WT, Kalappa N, Flores RH, Handwerger BS: Evidence for aerobic insufficiency in women with systemic Lupus erythematosus. Arthritis Rheum 49(1): 16-22, 2003.

8. Carvalho MR, Sato EI, Tebexreni AS, et al.: Effects of supervised cardiovascular training program on exercise tolerance, aerobic capacity, and quality of life in patients with systemic lupus erythematosus. Arthritis Rheum 53(6): 838-44, 2005.

9. Ayán C, Martín V: Systemic lupus erythematosus and exercise. Lupus 16(1): 5-9, 2007.

10. Ad Hoc Committee on Systemic Lupus Erythematosus Response Criteria for Fatigue. Measurement of fatigue in systemic lupus erythematosus: a systematic review. Arthritis Rheum 57(8): 1348-57, 2007.

11. Carvalho JF, Blank M, Kiss E, et al.: Anti-vitamin D, vitamin D in SLE: preliminary results. Ann N Y Acad Sci 1109: 550-7, 2007.

12. Kamen DL, Cooper GS, Bouali H, et al.: Vitamin D deficiency in systemic lupus erythematosus. Autoimmun Rev 5(2): 114-7, 2006.

13. Rahman P, Gladman DD, Urowitz MB: Smoking interferes with efficacy of antimalarial therapy in cutaneous lupus. J Rheumatol 25(9): 1716-9, 1998. 
14. Jewell ML, McCauliffe DP: Patients with cutaneous lupus erythematosus who smoke are less responsive to antimalarial treatment. J Am Acad Dermatol 42(6): 983-7, 2000.

15. Ghaussy NO, Sibbitt W Jr, Bankhurst AD, Qualls CR: Cigarette smoking and disease activity in systemic lupus erythematosus. J Rheumatol 30(6): 1215-21, 2003.

16. Westerweel PE, Luyten RK, Koomans HA, et al.: Premature atherosclerotic cardiovascular disease in systemic lupus erythematosus. Arthritis Rheum 2007;56(5): 1384-96.

17. Meinao IM, Sato EI, Andrade LE, et al.: Controlled trial with chloroquine diphosphate in systemic lupus erythematosus. Lupus 5: 237-41, 1996.

18. The Canadian Hydroxychloroquine Study Group: A randomized study of the effect of withdrawing hydroxychloroquine sulfate in systemic lupus erythematosus. N Engl J Med 324: 150-4, 1991.

19. Sachet JC, Borba EF, Bonfá E, et al.: Chloroquine increases low-density lipoprotein removal from plasma in systemic lupus patients. Lupus 16(4): 273-8, 2007.

20. Borba EF, Bonfa E: Long term beneficial effect of chloroquine diphosphate on lipoprotein profile in lupus patients with and without steroid therapy. J Rheumatol 28: 780-5, 2001.

21. Wallace DJ, Linker-Israeli M, Metzger AL, Stecher VJ: The relevance of antimalarial therapy with regard to thrombosis, hypercholesterolemia and cytokines in SLE. Lupus 2(Suppl 1): S13-5, 1993.

22. Kirou KA, Boumpas DT: Systemic glucocorticoid therapy in systemic lupus erythematosus. In: Wallace DJ, Hahn BH, editors. Dubois lupus erythematosus. 7th ed. Philadelphia: Lippincott Williams \& Wilkins; 2007. p. 1175-97.

23. Boumpas DT, Chrousos GP, Wilder RL, et al.: Glucocorticoid therapy for immune-mediated diseases: basic and clinical correlates. Ann Intern Med 119: 1198-208, 1993.

24. Maccune WJ, Marder WE, Riskalla M: Immunossuppressive drug therapy. In: Wallace DJ, Hahn BH, editors. Dubois lupus erythematosus. 7th ed. Philadelphia: Lippincott Williams \& Wilkins; 2007. p. 1198-224.

25. Carneiro JR, Sato EI: Double blind, randomized, placebo controlled clinical trial of methotrexate in systemic lupus erythematosus. J Rheumatol 26: 1275-9, 1999.

26. Walchner M, Messer G, Kind P: Phototesting and photoprotection in LE. Lupus 6: 167-74, 1997.

27. Vila LM, Mayor AM, Valentin AH, et al.: Association of sunlight exposure and photoprotection measures with clinical outcome in systemic lupus erythematosus. P R Health Sci J 18: 89-94, 1999.

28. Maggio KL, Singer MT, James WD: Clinical pearl: discoid lupus erythematosus - treatment with occlusive compression. J Am Acad Dermatol 35: 627-8, 1996.

29. Rangel LV, Santiago JM, Souza JCC, et al.: Terapia tópica com pimecrolimus em lesão cutânea refratária de lúpus eritematoso sistêmica. Rev Bras Reumatol 46: 230-3, 2006.

30. Luger T, Paul C: Potential new indications of topical calcineurin inhibitors. Dermatology 215 Suppl 1: 45, 2007.

31. Costner MI, Sontheimer RD, Provost TT: Lupus erythematosus. In: Sontheimer RD, Provost TT, editors. Cutaneous manifestations of rheumatic diseases. Philadelphia: Lippincott Williams \& Wilkins; 2003. p. 15-64.
32. Wenzel J, Brähler S, Bauer R, Tüting T: Efficacy and safety of methotrexate in recalcitrant cutaneous lupus erythematosus: results of a retrospective study in 43 patients. $\mathrm{Br} \mathrm{J}$ Dermatol 153: 157-62, 2005.

33. Callen JP, Spencer LV, Burruss JB, Holtman J: Azathioprine: an effective, corticosteroid-sparing therapy for patients with recalcitrant cutaneous lupus erythematosus or with recalcitrant cutaneous leukocytoclastic vasculitis. Arch Dermatol 127: 515-22, 1991.

34. Davis JC, Klippel JH: Antimalarials and immunossupressive therapies. In: Systemic Lupus Erythematosus. 4th ed. Lahita RG. 2004; p. 1279-81.

35. Bezerra ELM, Vilar MJP, Trindade Neto PB, Sato EI: Doubleblind, randomized, controlled clinical trial of clofazimine compared with chloroquine in patients with systemic lupus erythematosus. Arthritis Rheum 52: 3073-8, 2005.

36. Hall RP, Lawley TJ, Smith HR, Katz SI: Bullous eruption of systemic lupus erythematosus: dramatic response to dapsone therapy. Ann Intern Med 97: 165-70, 1982.

37. Cotell S, Robinson ND, Chan LS: Autoimmune blistering skin diseases. Am J Emerg Med 18: 288-99, 2000.

38. Atra E, Sato EI: Treatment of the cutaneous lesions of systemic lupus erythematosus with thalidomide. Clin Exp Rheumatol 11: 487-93, 1993.

39. Coelho A, Souto MI, Cardoso CR: Long-term thalidomide use in refractory cutaneous lesions in systemic lupus erythematosus: a 65 series of Brazilian patients. Lupus 14: 434-9, 2005.

40. Pisoni CN, Obermoser G, Cuadrado MJ: Skin manifestations of systemic lupus erythematosus refractory to multiple treatment modalities: poor results with mycophenolate mofetil. Clin Exp Rheumatol 23: 393-6, 2005.

41. Schanz S, Ulmer A, Rassner G: Successful treatment of subacute cutaneous lupus erythematosus with mycophenolate mofetil. Br J Dermatol 147: 174-8, 2002.

42. Horizon AA, Weisman MH: Nonsteroidals and salicylates for systemic lupus erythematosus. In: Wallace DJ, Hahn BH, editors. Dubois lupus erythematosus. 7th ed. Philadelphia: Lippincott Williams \& Wilkins; 2007. p. 1145-51.

43. Williams HJ, Egger MJ, Singer JZ, et al.: Comparison of hydroxychloroquine and placebo in the treatment of the arthropathy of mild systemic lupus erythematosus. J Rheumatol 21: 1457-62, 1994.

44. Rahman P, Humphrey-Murto S, Gladman DD, Urowitz MB: Efficacy and tolerability of methotrexate in antimalarial resistant lupus arthritis. J Rheumatol 25: 243-6, 1998.

45. Quismorio FP Jr: Hematologic and lymphoid abnormalities in systemic lupus erythematosus. In: Wallace DJ, Hahn BH, editors. Dubois lupus erythematosus. Philadelphia: Lippincott Williams \& Wilkins; 2007. p. 801-28.

46. Gomard-Menesson E, Ruivard M, Koenig M, et al.: Treatment of isolated severe immune hemolytic anaemia associated with systemic lupus erythematosus: 26 cases. Lupus 15: 223-31, 2006.

47. Jacob HS: Pulse steroids in hematologic diseases. Hosp Pract (Off Ed) 20: 87-94, 1985.

48. Pirofsky B: Immune haemolytic disease: the autoimmune haemolytic anaemias. Clin Haematol 4: 167-80, 1975. 
49. Aviña-Zubieta JA, Galindo-Rodriguez G, Robledo I, et al.: Long-term effectiveness of danazol,corticosteroids and cytotoxic drugs in the treatment of hematologic manifestations of systemic lupus erythematosus. Lupus 12: 52-57, 2003.

50. Alba P, Karim MY, Hunt BJ: Mycophenolate mofetil as a treatment for autoimmune haemolytic anaemia in patients with systemic lupus erythematosus and antiphospholipid syndrome. Lupus 12: 633-5, 2003.

51. Wang S-W, Cheng T-T: Systemic lupus erythematosus with refractory hemolytic anemia effectively treated with cyclosporin-A. Lupus 14: 483-5, 2005.

52. Pignon JM, Poirson E, Rochant H: Danazol in autoimmune haemolytic anaemia. Br J Haematol 83: 343-5, 1993.

53. Perotta S, Locatelli F, La Manna A, et al.: Anti-CD20 monoclonal antibody (rituximabe) for life-threatning autoimmune hemolytic anaemia in a patient with systemic lupus erythematosus. $\mathrm{Br} \mathrm{J}$ Haematol 116: 465-7, 2002.

54. Majer RV, Hyde RD: High-dose intravenous immunoglobulin in the treatment of autoimmune haemolytic anaemia. Clin Lab Haematol 10: 391-5, 1988.

55. Kondo H, Date Y, Sakai Y, et al.: Effective simultaneous rhGCSF and methylprednisolone pulse therapy in agranulocytosis associated with systemic lupus erythematosus. Am J Hematol 46: 157-8, 1994.

56. Euler HH, Harten P, Zeuner RA, et al.: Recombinant human granulocyte colony stimulating factor in patients with systemic lupus erythematosus associated neutropenia and refractory infections. J Rheumatol 24: 215-37, 1997.

57. Cervera H, Jara LJ, Pizarro S, et al.: Danazol for systemic lupus erythematosus with refractory autoimmune thrombocytopenia or Evans syndrome. J Rheumatol 22: 1867-71, 1995.

58. Roach BA, Hutchinson GJ: Treatment of refractory, systemic lupus erythematosusassociated thrombocytopenia with intermittent low-dose intravenous cyclophosphamide. Arthritis Rheum 36: 682-4, 1993.

59. Arnal C, Piette JC, Leone J, et al.: Treatment of severe immune thrombocytopenia associated with systemic lupus erythematosus: 59 cases. J Rheumatol 29: 75-83, 2002.

60. Goebel KM, Gassel WD, Goebel FD: Evaluation of azathioprine in autoimmune thrombocytopenia and lupus erythematosus. Scand J Haematol 10: 28-34, 1973.

61. Vasoo S, Thumboo J, Fong KY: Refractory immune thrombocytopenia in systemic lupus erythematosus: response to mycophenolate mofetil. Lupus 12: 630-2, 2003.

62. Quartucio L, Sacco S, Franzolini N, et al.: Efficacy of cyclosporin-A in the long-term management of thrombocytopenia associated with systemic lupus erythematosus. Lupus 15: 76-9, 2006.

63. Shanafelt TD, Madueme HL, Wolf RC, et al.: Rituximab for immune cytopenia in adults: idiopathic thrombocytopenic purpura,autoimmune hemolytic anemia,and Evans syndrome. Mayo Clin Proc 78: 1340-6, 2003.

64. Ten Cate R, Smiers FJ, Bredius RG, et al.: Anti-CD20 monoclonal antibody(rituximab) for refractory autoimmune thrombocytopenia in a girl with systemic lupus erythematosus. Rheumatology (Oxford) 43; 244, 2004.
65. Ter Borg EJ, Kallenberg CG: Treatment of severe thrombocytopenia in systemic lupus erythematosus with intravenous gammaglobulin. Ann Rheum Dis 51: 1149-51, 1992.

66. Chandramouli NB, Rodgers GM: Prolonged immunoglobulin and platelet infusion for treatment of immune thrombocytopenia. Am J Hematol 65: 85-6, 2000.

67. You NY, Tefferi A, Nagorney DM: Outcome of splenectomy for thrombocytopenia associated with systemic lupus erythematosus. Ann Surg 240: 286-96, 2004.

68. Maksimowicz-McKinnon K, Manzi S: Cardiovascular manifestations of lupus. In: Wallace DJ, Hahn BH, editors. Dubois lupus erythematosus. Philadelphia: Lippincott Williams \& Wilkins; 2007. p. 663-77.

69. Westerweel PE, Luyten RK, Koomans HA, et al.: Premature atherosclerotic cardiovascular disease in systemic lupus erythematosus. Arthritis Rheum. 2007; 56(5): 1384-96.

70. Manzi S, Meilahn EN, Rairie JE, et al.: Age-specific incidence rates of myocardial infarction and angina in women with systemic lupus erythematosus: comparison with the Framingham Study. Am J Epidemiol 145(5): 408-15, 1997.

71. Petri M, Lakatta C, Magder L, Goldman D: Effect of prednisone and hydroxychloroquine on coronary artery disease risk factors in systemic lupus erythematosus: a longitudinal data analysis. Am J Med 96: 254-9, 1994.

72. Petri M, Kiani AN, Post W, Magder L: Lupus Atherosclerosis Preventio Study (LAPS): a randomized double blind placebo controlled trial of atorvastatin versus placebo. Arthritis Rheum 54 Suppl: S520, 2006.

73. Ferreira GA, Navarro TP, Telles RW, et al.: Atorvastatin therapy improves endothelial-dependent vasodilation in patients with systemic lupus erythematosus: an 8 weeks controlled trial. Rheumatology (Oxford) 46(10): 1560-5, 2007.

74. D'Cruz D, Khamashta MA, Hughes G: Pulmonary manifestations of systemic lupus erythematosus. In: Wallace DJ, Hahn BH. Dubois' Lupus Erythematosus. Philadelphia, 7th ed. Lippincott Williams \& Wilkins; 2007. p. 678-99.

75. Man BL, Mok CC: Serositis related to systemic lupus erythematosus: prevalence and outcome. Lupus 14: 822-6, 2005.

76. Kao AH, Manzi S: How to manage patients with cardiopulmonary disease? Best Pract Res Clin Rheumatol 16: 211-27, 2002.

77. McKnight KM, Adair NE, Agudelo CA: Successful use of tetracycline pleurodesis to treat massive pleural effusion secondary to systemic lupus erythematosus. Arthritis Rheum 34: 1483-4, 1991.

78. Schwab EP, Schumacher HR Jr, Freundlich B, Callegari PE: Pulmonary alveolar hemorrhage in systemic lupus erythematosus. Semin Arthritis Rheum 23: 8-15, 1993.

79. Weinrib L, Sharma OP, Quismorio FP Jr: A long-term study of interstitial lung disease in systemic lupus erythematosus. Semin Arthritis Rheum 20: 48-56, 1990.

80. Okada M, Suzuki K, Matsumoto M, et al.: Intermittent intravenous cyclophosphamide pulse therapy for the treatment of active interstitial lung disease associated with collagen vascular diseases. Mod Rheumatol 17: 131-6, 2007.

81. Heresi GA, Minai OA: Lupus-associated pulmonary hypertension: long-term response to vasoactive therapy. Respir Med 101: 2099-107, 2007. 
82. Denton CP, Humbert M, Rubin L, Black CM: Bosentan treatment for pulmonary arterial hypertension related to connective tissue disease: a subgroup analysis of the pivotal clinical trials and their open-label extensions. Ann Rheum Dis 65: 1336-40, 2006.

83. Galiè N, Manes A, Farahani KV, et al.: Pulmonary arterial hypertension associated to connective tissue diseases. Lupus 14: 713-7, 2005.

84. Sanchez O, Sitbon O, Jaï X, et al.: Immunosuppressive therapy in connective tissue diseases-associated pulmonary arterial hypertension. Chest 130: 182-9, 2006.

85. The American College of Rheumatology nomenclature and case definitions for neuropsychiatric lupus syndromes. Arthritis Rheum 42: 599-608, 1999.

86. Boumpas DT, Yamada H, Patronas NJ, et al.: Pulse cyclophosphamide for severe neuropsychiatric lupus. Q J Med 81: 975-84, 1991.

87. West SG: The nervous system. In: Wallace DJ, Hahn BH. Dubois' Lupus Erythematosus. Philadelphia. 7th ed. Lippincott Williams \& Wilkins; 2007. p. 707-46.

88. Barile L, Lavalle C: Transverse myelitis in systemic lupus erythematosus - the effect of IV pulse methylprednisolone and cyclophosphamide. J Rheumatol 19: 370-2, 1992.

89. Tokunaga M, Saiko K, Kawabata D, et al.: Efficacy of rituximab (anti-CD20) for refractory systemic lupus erythematosus involving the central nervous system. Ann Rheum Dis 66: 470-5, 2007.

90. Dooley MA: Clinical and laboratory features of lupus nephritis. In: Wallace DJ, Hahn BH. Dubois' Lupus Erythematosus. Philadelphia, 7th ed. Lippincott Williams \& Wilkins; 2007. p. 1112-30.

91. Grande JP, Ballow JE: Renal biopsy in lupus nephritis. Lupus 7: 611-7, 1998.

92. Weening JJ, D'Agati VD, Schwartz MM, et al.: The classification of glomerulonephritis in systemic lupus erythematosus revisited. J Am Soc Nephrol 15(2): 241-50, 2004.

93. Weening JJ, D'Agati VD, Schwartz MM, et al.: The classification of glomerulonephritis in systemic lupus erythematosus revisited. Kidney Int. 65(2): 521-30, 2004.

94. Renal disease subcommitee of the ACR Ad Hoc Committee on SLE response criteria: The American College of Rheumatology response criteria for proliferative and membranous renal disease in SLE clinical trials. Arthritis Rheum 54: 421-32, 2006.

95. Ruggenenti P, Perna A, Gherardi G, et al.: Renal function and requirement for dialysis in chronic nephropathy patients on long-term ramipril: REIN follow-up trial. Gruppo Italiano di Studi Epidemiologici in Nefrologia (GISEN). Ramipril Efficacy in Nephropathy. Lancet 352: 1252-6, 1998.

96. Praga M: Slowing the progression of renal failure. Kidney Int Suppl 80: 18-22, 2002.

97. Pollak VE: Treatment of lupus nephritis. Nippon Jinzo Gakkai Shi 25: 776-85, 1983.

98. Kimberly RP, Lockshin MD, Sherman RL, et al.: High-dose intravenous methylprednisolone pulse therapy in systemic lupus erythematosus. Am J Med 70: 817-24, 1981.

99. Austin HA 3rd, Klippel JH, Balow JE, et al.: Therapy of lupus nephritis: controlled trial of prednisone and cytotoxic drugs. $\mathrm{N}$ Engl J Med 314: 614-9, 1986.
100. Balow JE: Choosing treatment for proliferative lupus nephritis. Arthritis Rheum 46: 1981-3, 2002.

101. Flanc RS, Roberts MA, Strippoli GF, et al.: Treatment of diffuse proliferative lupus nephritis: a meta-analysis of randomized controlled trials. Am J Kidney Dis 43: 197-208, 2004.

102. Bansal VK, Beto JA: Treatment of lupus nephritis: a metaanalysis of clinical trials. Am J Kidney Dis 29: 193-9, 1997.

103. Chan TM, Li FK, Tang CS, et al.: Efficacy of mycophenolate mofetil in patients with diffuse proliferative lupus nephritis. Hong Kong-Guangzhou Nephrology Study Group. N Engl J Med 343: 1156-62, 2000.

104. Houssiau FA, Vasconcelos C, D'Cruz D, et al.: Immunosuppressive therapy in lupus nephritis: the Euro-Lupus Nephritis Trial, a randomized trial of low-dose versus high-dose intravenous cyclophosphamide. Arthritis Rheum 46: 2121-31, 2002.

105. Contreras G, Pardo V, Leclercq B, et al.: Sequential therapies for proliferative lupus nephritis. N Engl J Med 350: 971-80, 2004.

106. Ginzler EM, Aranow C, Buyon J, et al.: A multicenter study of MMF Vs IV cyclophosphamide as induction therapy for severe lupus nephritis: preliminary results. N Eng J Med 353: 2219-28, 2005.

107. Sinclair A, Appel G, Dooley MA, et al.: Mycophenolate mofetil as induction and maintenance therapy for lupus nephritis: rationale and protocol for the randomized, controlled Aspreva Lupus Management Study (ALMS). Lupus. 16(12): 972-80, 2007.

108. Kolasinski SL, Chung JB, Albert DA: What do we know about lupus membranous nephropathy? An analytic review. Arthritis Rheum 47: 450-5, 2002.

109. Chan TM, Li FK, Hao WK, et al.: Treatment of membranous lupus nephritis with nephrotic syndrome by sequential immunosuppression. Lupus 8: 545-51, 1999.

110. Radhakrishnan J, Kunis CL, D'Agati V, Appel GB: Cyclosporine treatment of lupus membranous nephropathy. Clin Nephrol 42: 147-54, 1994.

111. Mok CC, Ying KY, Lau CS, et al.: Treatment of pure membranous lupus nephropathy with prednisone and azathioprine: an openlabel trial. Am J Kidney Dis 43: 269-76, 2004.

112. Borba EF, Guedes LK, Christmann RB, et al.: Mycophenolate mofetil is effective in reducing lupus glomerulonephritis proteinuria. Rheumatol Int. 26(12): 1078-83, 2006.

113. Sabahi R, Anolik JH: B-cell-target for SLE. Drugs 66(15): 1933-48, 2006.

114. Bhat P: B lymphocytes and lupus nephritis: new insights into pathogenesis and targeted therapies. Kidney Int 73: 261-8, 2008.

115. Stone JH: End-stage renal disease in lupus: disease activity, dialysis, and the outcome of transplantation. Lupus 7: 654-9, 1998.

116. Urowitz MB, Gladman DD, Farewell VT, et al.: Lupus and pregnancy studies. Arthritis Rheum 36: 1392-7, 1993.

117. Clowaw ME, Magder LS, Petri M: The impact of increased lupus activity on obstetric outcomes. Arthritis Rheum 52: 514-21, 2005.

118. Sánchez-Guerrero J, Uribe AG, Jiménez-Santana L, et al.: A trial of contraceptive methods in women with Systemic lupus erythematosus. N Engl J Med 353: 2539-49, 2005. 
119. Petri M, Kim MY, Kalunian KC, et al.: OC-SELENA Trial. Combined oral contraceptives in women with systemic lupus erythematosus. N Engl J Med 353: 2550-8, 2005.

120. Buyon JP, Kalunian KC, Ramsey-Goldman R, et al.: Assessing disease activity in SLE patients during pregnancy. Lupus 8: 677-84, 1999.

121. Petri M: Pregnancy in SLE. In: Hochberg M, ed. Rheumatology. 3th. ED; 2003. p. 1427-30.

122. Wilson WA, Gharavi AE, Koike T, et al.: International consensus statement on preliminary classification criteria for definite antiphospholipid syndrome: report of an international workshop. Arthritis Rheum 42: 1309-11, 1999.

123. Buyon JP, Clancy RM: Neonatal lupus syndromes. Curr Opin Rheumatol 15: 535-41, 2003.

124. Cervera R, Font J, Carmona F, Balasch J: Pregnancy outcome in systemic lupus erythematosus: good news for the new millennium. Autoimmun Rev 1: 354-9, 2002.

125. Anderson PO: Drugs and breast feeding. Semin Perinatol 3:271-8, 1979.

126. Bermas BL, Hill JA: Effects of immunosuppressive drugs during pregnancy. Arthritis Rheum 38: 1722-32, 1995.

127. Levy RA, Vilela VS, Cataldo MJ, et al.: Hydroxychloroquine in lupus pregnancy: double-blind and placebo-controled study. Lupus 10: 401-4, 2001.

128. Ost L, Wettrell G, Bjorkhem I, Rane A: Prednisolone excretion in human milk. J Pediatr 106: 1008-11, 1985.

129. Petri M: Hydroxychloroquine use in Baltimore Lupus Cohort: effects on lipids, glucose and thrombosis. Lupus 5(Suppl 1): S16-22, 1996

130. Bertolaccini ML, Khamashta MA: Laboratory diagnosis and management challenges in the antiphospholipid syndrome. Lupus 15: 172-8, 2006.

131. Jungers P, Dougados M, Pélissier C, et al.: Influence of oral contraceptive therapy on the activity of systemic lupus erythematosus. Arthritis Rheum 25(6): 618-23, 1982.

132. Mintz G, Gutiérrez G, Delezé M, Rodríguez E: Contraception with progestagens in systemic lupus erythematosus. Contraception 30(1): 29-38, 1984

133. Buyon JP, Petri MA, Kim MY, et al.: The effect of combined estrogen and progesterone hormone replacement therapy on disease activity in systemic lupus erythematosus: a randomized trial. Ann Intern Med 142: 953-62, 2005

134. Sánchez-Guerrero J, González-Pérez M, Durand-Carbajal M, et al.: Menopause hormonal therapy in women with systemic lupus erythematosus. Arthritis Rheum 56(9): 3070-9, 2007.
135. Lahita RG: Hormonal contraception and replacement and the use of androgens in the antiphospholipid syndrome. J Autoimmun 15: 213-6, 2000.

136. Klippel JH, Karsh J, Stahl NI, et al.: A controlled study of pneumococcal polysaccharide vaccine in systemic lupus erythematosus. Arthritis Rheum 22: 1321-5, 1979.

137. Abu-Shakra M, Zalmanson S, Neumann L, et al.: Influenza virus vaccination of patients with systemic lupus erythematosus: effects on disease activity. J Rheumatol 27: 1681-5, 2000.

138. O'Neill SG, Isenberg DA: Immunizing patients with systemic lupus erythematosus: a review of effectiveness and safety. Lupus 15(11): 778-83, 2006

139. Kuruma KA, Borba EF, Lopes MH, et al.: Safety and efficacy of hepatitis B vaccine in systemic lupus erythematosus. Lupus 16(5): 350-4, 2007

140. Liang MH, Fortin PR, Isenberg DA, Snaith L: Quantitative clinical assessment of disease activity in systemic lupus erythematosus: progress report and research agenda. Rheumatol Int 11: 133-6, 1991.

141. Strand V, Gladman D, Isenberg D, et al.: Outcome measures to be used in clinical trials in systemic lupus erythematosus. J Rheumatol 26: 490-7, 1999.

142. Strand V, Gladman D, Isenberg D, et al.: Endpoints: consensus recommendations from OMERACT IV. Lupus 9: 322-7, 2000 .

143. Bombardier C, Gladman DD, Urowitz MB, et al.: Derivation of the SLEDAI. A disease activity index for lupus patients. The Committee on Prognosis Studies in SLE. Arthritis Rheum 35(6): 630-40, 1992.

144. Gladman DD, Ibañez D, Urowitz MB: Systemic lupus erythematosus disease activity index 2000. J Rheumatol 29: 288-91, 2002

145. Machado C, Ruperto N: Consenso em Reumatologia Pediátrica Parte II - definição de melhora clínica para o lúpus eritematoso sistêmico e dermatomiosite juvenil. Rev Bras Reumatol 45 (1): $14-9,2005$

146. Gladman D, Ginzler E, Goldsmith C, et al.: The development and initial validation of the Systemic Lupus International Collaborating Clinics/American College of Rheumatology damage index for systemic lupus erythematosus. Arthritis Rheum 39(3): 363-9, 1996.

147. Gladman DD, Goldsmith CH, Urowitz MB, et al.: The Systemic Lupus International Collaborating Clinics/American College of Rheumatology (SLICC/ACR) Damage Index for Systemic Lupus Erythematosus International Comparison. J Rheumatol 27(2): 373-6, 2000. 